\title{
Chance Discovery in Credit Risk Management: Estimation of Chain Reaction Bankruptcy Structure by Directed KeyGraph
}

\author{
Shinichi Goda and Yukio Ohsawa \\ School of Engineering, The University of Tokyo, 113-8656 Japan \\ sgoda@mwa.biglobe.ne.jp
}

\begin{abstract}
Credit risk management based on portfolio theory becomes popular in recent Japanese financial industry. But consideration and modeling of chain reaction bankruptcy effect in credit portfolio analysis leave much room for improvement. That is mainly because method for grasping relations among companies with limited data is underdeveloped. In this article, chance discovery method with directed KeyGraph is applied to estimate industrial relations that are to include companies' relations that transmit chain reaction of bankruptcy. The steps for the data analysis are introduced and result of example analysis with default data in Kyushu, Japan, 2005 is presented.
\end{abstract}

Keywords: chance discovery, credit risk, chain reaction, bankruptcy.

\section{Introduction}

Credit risk management based on portfolio theory becomes popular in recent Japanese financial industry promoted by introduction of BIS regulation and increasing use of model-based loan decision making. Simulation method comes to be common tool for analysis of credit portfolio and simulation models have been developed for credit risk management [1/234]. However, there still remain major areas for improvement. Analysis on chain reaction bankruptcies is one of these areas.

\section{Effect of Chain Reaction Bankruptcy}

Chain reaction bankruptcies are common phenomenon. Its general definition is like "bankruptcy triggered by a preceding default of a company that has trade and other relations with the bankrupting company".

Most of industry experts regard it necessary to take the effect of chain reaction bankruptcy into accounts when they analyze profile of their credit risk portfolio. By introducing chain reaction factor into analysis, we can expect to better grasp the risk profile of credit risk portfolio since amount of loss caused by a default can be larger if there are other bankruptcies triggered by the default. 
However, majority of simulation models actually used in business do not fully take the chain reaction into account. That is mainly because it is difficult to directly grasp relations among companies since available data is limited as;

1. Background information about bankruptcies publicly available is very limited, and it is not organized in way that can be used for statistical analysis.

2. Corporate data related to relations among corporation is very limited to public. Few companies make trade, financial and technological relations public, but very few with numbers.

\section{Current Methods to Grasp Relations}

Adjustments have been devised in models and simulators to include relation effect, but there is much room for improvement. A major method to take the effect into account is to grasp relations among companies by measuring co-relations among movements of the security price of the companies (In this method, a company's security price is regarded as representative of default probability of the company). But this method is applicable only to security issuing large companies whose number is very small. On the other hand, most of companies in a credit risk portfolio are non-security-issuing and small-mid size.

Another way is to make industry groups represent companies that belong to them, and to estimate relations among companies by grasping relations among the industries. Co-relations are measured among indexes of securities issued by companies in the industry groups and are applied to non-security-issuing ones in the groups. But the idea to estimate relations among industries with co-relations among security prices seems to be unreasonable. The most of Japanese companies are non-security-issuing ones and there is not enough evidence of the relations among security-issuing companies being same as that of non-issuing ones.

\section{Grasp Chain Reaction Bankruptcy Structure by Chance Discovery Method}

We proposed a method that detects relationship among bankrupted companies, without direct information of trade relations i.e., by chance discovery method in our previous work 5]. (The basic idea of this method follows the method that used for the chance discovery of earthquake by Ohsawa, Y. 6 67).)

In this method, we estimate trade and/or other relations among companies defaulted in a geographical area within a certain time period, by visualizing relations among industry groups that include the defaults with KeyGraph. The method is based on the assumptions as follows;

1. There should be relations that transmitted factors causing defaults among companies that defaulted in a geographical area within a certain time period.

2. The default transmitting relations among companies should be mainly based on and represented by relations among industry groups. As seen in the above 
definition of chain reaction bankruptcy, "trade relation" is generally regarded as one of the most influential relations. Trade relation between a pair of industry is generally universal among companies in the paired industries.

3. Default transmitting relations among industries could be paths to transmit default of a company in an industry to other companies in other industries.

Suppose that cloth retailer A and cloth wholesaler B defaulted within a month successively in Kansai district. Suppose other sets of cloth retailer and wholesaler those located in same districts defaulted successively within a month repeatedly. We can estimate with high confidence that there were sets of trade relation between the cloth retailer and the wholesaler defaulted successively and that the sets of trade relation between the two companies caused the successive default, even if there is no public information of sets of trade relations between the two companies. We can estimate so based on expert's knowledge about cloth trading industry and on the observed default patterns analyzed with KeyGraph.

\section{Methodology}

\subsection{Original Method}

First, we explain the original method proposed in our previous work [5] and introduce its basic idea. Steps are as follows (see Table 1);

Step1. Preparation of data

1. Data of defaults: each default event has attributes of default date, geographical area in which the defaulted company is located and an industry to which it belongs.

2. Sorting: group the defaults by area and sort the events in each area by default dates.

3. Select companies that seemed to have triggered chain reaction.

Step2. Transformation of company data to industry data

Transform company data prepared in Step1. to industry data by replacing a company's name to a code of an industry to which the company belongs.

\section{Step3. Transformation of data to sentence form}

1. Make the default events grouped by area in sentence form by order of their default dates. Each event is denoted by industry name and spaced.

2. Form one sentence starting from a triggering company and ending at a company whose default date is after the default date of the starting company.

Step4. Discovery of relations among industries by KeyGraph

1. Extract co-occurrence of default events by using KeyGraph with sentences formed default data. 
2. Interpret a result of KeyGraph and estimate relations among industries. It is important to read relations expressed in a KeyGraph with experts' knowledge.

Examples of experts' knowledge about factors that are supposed to work behind relations extracted by KeyGraph are as listed below.

a. Technological and business relations among industries

(example) An automobile is made of steel, glass, tires and electronic parts.

b. Commonality of customers among industries

(example) Consumers living in Kyushu region shop at cloth/food retailers and eat restaurant both located in Kyushu.

c. Ownership relation:

Table 1. Example of data and sentence

Step2. Data (2) grouped by area,

Step1. Data (1) sorted by date

\begin{tabular}{|c|c|c|c|c|}
\hline No. & $\begin{array}{c}\text { date of } \\
\text { default }\end{array}$ & comp. & ind. code & area \\
\hline 1 & $2005 / 1 / 4$ & A & 101 & north \\
\hline 2 & $2005 / 1 / 15$ & B & 210 & south \\
\hline 3 & $2005 / 1 / 30$ & C & 303 & east \\
\hline & $\vdots$ & & & \\
\hline N & $2005 / 12 / 28$ & ZZZ & 330 & north \\
\hline
\end{tabular}
and sorted by date

Step3. Transform to sentence form

Series starting from D1 = $101110418 \ldots$

Series starting from D2 = $210105330 \ldots$

Series starting from D3 = $303240440 \ldots$

\begin{tabular}{|c|c|c|c|c|}
\hline No. & $\begin{array}{c}\text { and sorted by date } \\
\text { date of } \\
\text { default }\end{array}$ & comp. & $\begin{array}{c}\text { ind. } \\
\text { code }\end{array}$ & area \\
\hline 3 & $2005 / 1 / 30$ & $\mathrm{C}$ & 303 & east \\
\hline 8 & $2005 / 3 / 20$ & $\mathrm{H}$ & 440 & east \\
\hline & $\vdots$ & & & \\
\hline 1 & $2005 / 4 / 1$ & $\mathrm{~A}$ & 101 & north \\
\hline & $\vdots$ & & & \\
\hline $\mathrm{N}$ & $2005 / 12 / 28$ & $\mathrm{ZZZ}$ & 330 & north \\
\hline & $\vdots$ & & & \\
\hline 9 & $2005 / 3 / 29$ & $\mathrm{I}$ & 211 & west \\
\hline
\end{tabular}

\subsection{Time Order Method (with Directed KeyGraph)}

The original method had two points to be improved;

1. Time order among defaults is not captured. That makes the estimation of causal relation among defaults difficult.

2. The criteria for selecting trigger defaults were not clear enough.

In our previous work [5], we made a sentence with default events in a month that starts from a hypothetical trigger default that were selected from a list of defaults that was made by Japanese SME Agency, based on the size or impact of defaults.

We newly introduce time order method to deal with above points. The basic idea of the new method is to try to better distinguish the causal relations among defaults with the time order among them. 
Table 2. Method for making time ordered pair of defalts

\begin{tabular}{|c|c|c|c|c|c|c|c|c|c|c|c|c|}
\hline \multicolumn{2}{|c|}{ Default date } & $1 / 1$ & $1 / 10$ & $1 / 20$ & $2 / 1$ & $2 / 5$ & $2 / 25$ & $2 / 28$ & & $12 / 1$ & $12 / 20$ & $12 / 30$ \\
\hline \multicolumn{2}{|c|}{ Name of company } & $\mathrm{A}$ & $\mathrm{B}$ & $\mathrm{C}$ & $\mathrm{D}$ & $\mathrm{E}$ & $\mathrm{F}$ & $\mathrm{G}$ & $\cdots$ & $\mathrm{X}$ & $\mathrm{Y}$ & $\mathrm{Z}$ \\
\hline \multicolumn{2}{|c|}{ Industry code } & 10 & 20 & 30 & 40 & 10 & 30 & 20 & & 40 & 10 & 50 \\
\hline \multirow[t]{8}{*}{ Pair } & Start from 10(A) & & S10_20 & S10_30 & & & & & & & & \\
\hline & Start from 20(B) & & & S20_30 & S20_40 & S20_10 & & & & & & \\
\hline & Start from $30(\mathrm{C})$ & & & & $\bar{S} 30 \_40$ & S30_10 & & & & & & \\
\hline & Start from $40(\mathrm{D})$ & & & & & S40_10 & S40_30 & S40_20 & & & & \\
\hline & Start from $10(\mathrm{E})$ & & & & & & S10_30 & S10_20 & $\cdots$ & & & \\
\hline & $\cdots$ & & & & & & & & & $\cdots$ & & \\
\hline & Start from $20(\mathrm{~W})$ & & & & & & & & & $\mathrm{S} 20 \_40$ & S20_10 & \\
\hline & Start from $40(\mathrm{X})$ & & & & & & & & & & S40_10 & S40_50 \\
\hline
\end{tabular}

With time order method, by making a sentence include only a pair of defaults with distinction of earlier event of the two, the time order among defaults can be expressed by KeyGraph with direction - we name it as "directed KeyGraph". Detailed steps are as follows (see Table 2);

1. Make a sentence of a pair of defaults.

2. Put "S" to industry code of the earlier default in a pair to distinguish it as a starting default.

3. Make series of pairs with a selected starting default indicated by "S", and ending defaults each of which follows the starting default within a period.

4. Select another default that occurred next to the first default as the second starting event and take step 3.

5. Repeat step 3. and 4. until all the defaults in the analyzed period are selected as starting events.

6. Make and include linking pairs to link the nodes those are captured as ending events but at the same time are starting ones. For example, in Table 2, default E, that is coded as 10, is included in pair "S20_10", "S30_10", "S40_10" as an ending default and also included in pair "S10_30", "S10_20" as a starting one.

When the paired data is analyzed with directed KeyGraph, starting defaults are indicated by "S" put on the industry code. When a node, "S20" for example, is linked to another node, "10", it means that defaults in the industry 20 occurred before defaults in 10, indicating that defaults in 20 trigger the defaults in 10 . In case two nodes of same industry are linked, like "10 - S10", it means ending defaults in "10" are at the same time starting defaults.

\section{Case Study - Analysis of Chain Reaction Structure of Bankruptcies in Kyushu, 2005}

As a case study, we applied time order method described above to data of bankruptcies in Kyushu district, Japan, 2005. The reason for limiting area for analysis only to Kyushu district is simply to make the size of data controllable. 
A. Contents of data

Samples are 343 defaults in Kyushu, a local part of the Japanese samples described below. About 10,400 pairs are made from the above selected 343 default samples.

1. Japanese samples, about 3,400 defaults, are randomly selected from the all defaulted companies that defaulted based on bankruptcy laws and published in the official gazette in Japan, 2005. The area consists of 9 districts including Kyushu. Samples are categorized in about 200 mid-level industry groups by author, based on the industry categories defined by Teikoku Databank, Ltd.

2. Period for pairing one starting default with ending ones is set to one month.

3. About 6,200 linking pairs are added.

\section{B. Analysis}

First, the data was analyzed by original method and then by time order method.

\section{(1) Original method (see Fig. 1 for result)}

With this KeyGraph by original method, we can understand that defaults in linked industries occurred in a close time period. But it is difficult to estimates whether the co-occurrence is based on chain reaction. That is partly because this graph lacks of information about causal relation among defaults.

\section{(2) Time order method with directed KeyGraph (see Fig. 2 for result)}

In Fig. 2, time orders among defaults in the industries are shown by arrows indicating causal relations among them. Nodes consist of starting defaults are indicated by "S_" put on industry codes. Time order is expressed by arrows from a node with "S_" to another node without "S_." Circled pair of two nodes, one with "S_", are in same industry. For example, an arrow from S_C61 goes to C63, showing defaults in C61 occurred before defaults in C63 in major case, not vice versa. C63 is linked to S_C63 and two nodes are circled. An arrow then goes from S_C63 to C62. It shows that defaults in C63 those occurred after defaults in C61 are, at the same time, defaults those occurred before those in C62. It indicates that defaults in C63 caused by those in C61 then triggered defaults in C62.

When we see Fig. 2 this way, we can better estimate causal relations among defaults. The number of arrows go out from C61 (=S_C61) and C62 (=S_C62) are greater than those go into $\mathrm{C} 61$ and $\mathrm{C} 62$, that indicates defaults in civil engineering/construction and civil engineering industry caused defaults in other industries in major case, not vice versa. The defaults in C61, C62 might then trigger defaults in variety of related industries like C54 (brick layer work), C77 (pipe work), T31 (transportation_trade), i.e. Arrows from S_W03 (textile/cloth/others wholesale) go to C62 and to C63, indicating defaults in W03, caused by depressed consumer spending for cloth, were source of defaults in C62 and in C63 other than decreased public construction work. Many arrows go out from R39, indicating defaults of super markets, caused by depressed consumer spending, triggered defaults of groceries, toy/accessory shops in the super markets and of electric machinery wholesalers who trade with the market. 
$\mathrm{JaJa}[20-20-20-15]$

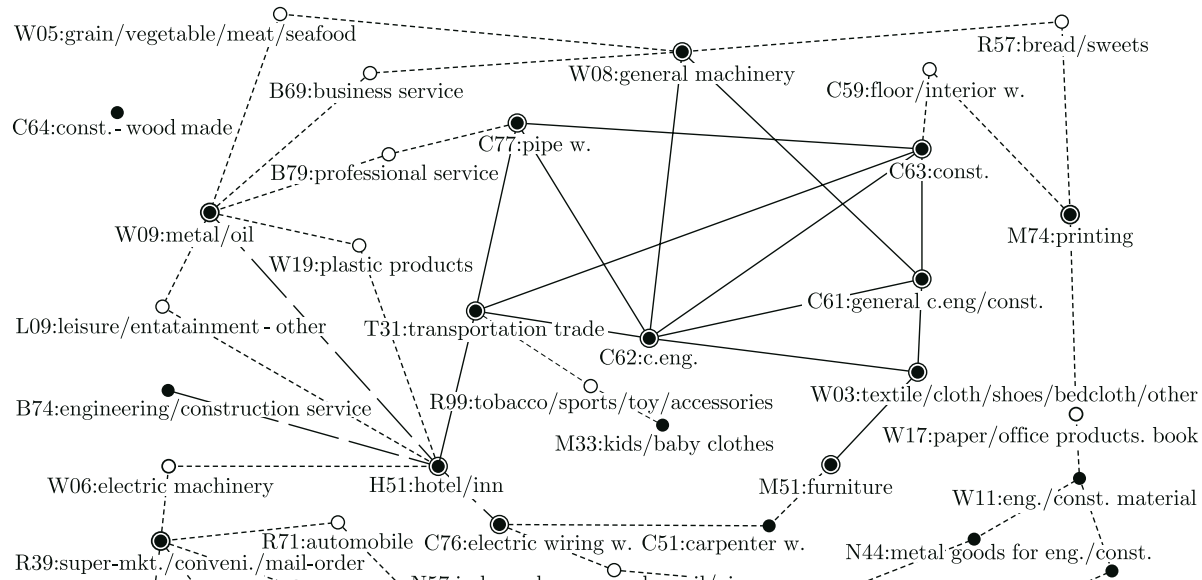

R39:super-mkt./conveni./mail-order

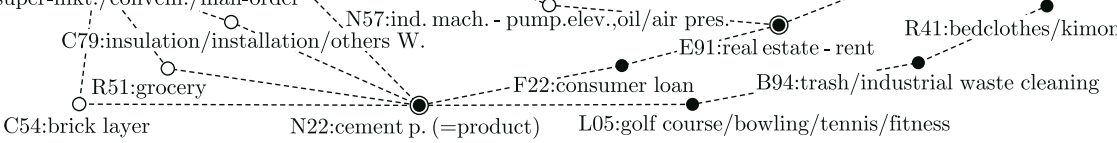

(C:civil engineering/construction, M, N: manufacturing, W: wholesale, R: retail, T: transportation, E: real estate)

Fig. 1. KeyGraph by original method

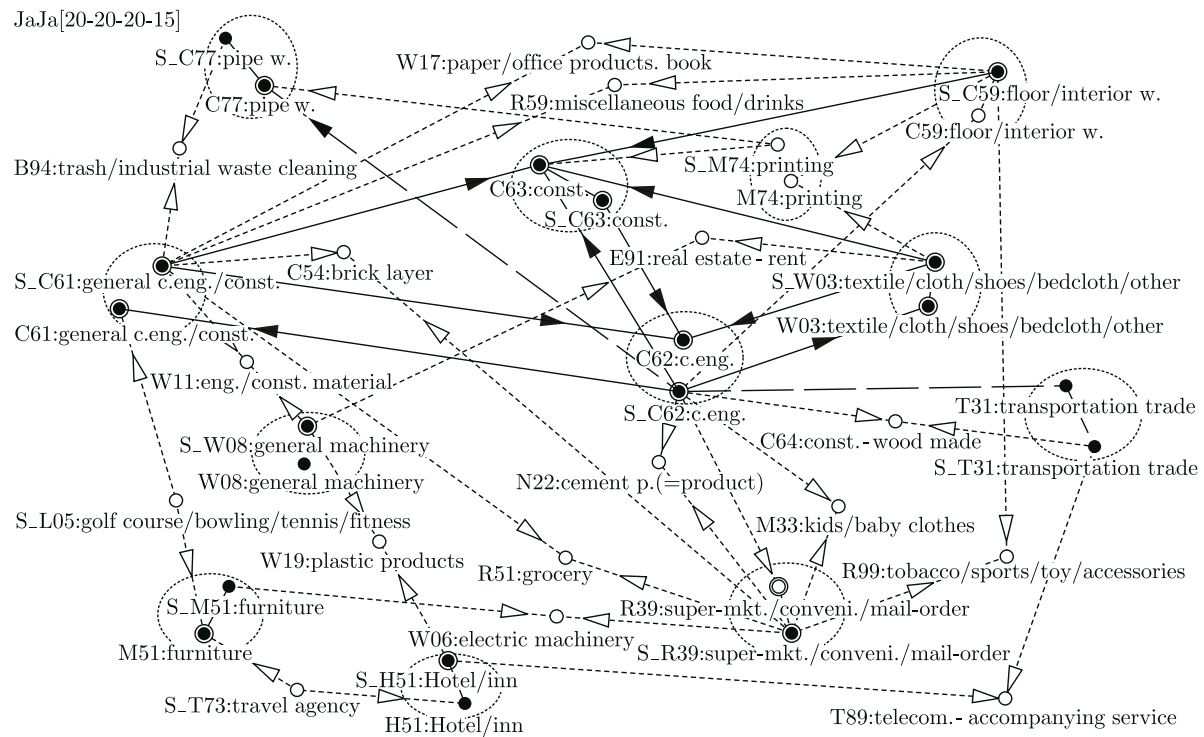

(C:civil engineering/construction, $\mathrm{M}, \mathrm{N}$ : manufacturing, W: wholesale, R: retail, T: transportation, E: real estate)

Fig. 2. KeyGraph by time order method ("S_" indicates starting default) 


\section{Conclusion}

In this article, we applied chance discovery method to estimate structure of industrial relations that are to transmit chain reaction of bankruptcy. We introduced time order method with directed KeyGraph to capture/express time order of defaults and to better estimate causal relations among defaults. The result of the analysis of default data in Kyushu 2005 was promising. With further accumulation of analyses and improvement of method, a structure estimated by chance discovery method will sufficiently be a base for risk analysis and risk management of a credit portfolio.

The areas for further improvements are;

1. techniques for extracting appropriate time range between start/end of defaults

2. measurement of influence over a default probability of an industry/company of a default event to be transmitted through estimated industrial relations

3. modeling of estimated industrial relations in network structure for the use for risk analysis and risk management of a credit portfolio

\section{References}

1. Saunders, A.: Credit Risk Measurement (Japanese), Kinyu Zaisei Jijo Press, 2001.

2. FISC: "Working Report on Risk Management Model", 1999.

3. Nakabayashi, A. and Sasaki, M.: "Models for credit risk measurement and its application to Japanese bank", FRI Review, 2 No. 2, 1998.

4. Torii, H.: "Portfolio based credit risk management models - its effectiveness and limit", Weekly Kinyu Zaisei Jijo Magazine, June, 1998.

5. Goda, S. and Ohsawa, Y.: "Chance Discovery in Credit Risk Management - Estimation of chain reaction bankruptcy structure by chance discovery method", Proceedings of the 2006 IEEE International Conference on Systems, Man, and Cybernetics (6 Volumes), October 8-11, 2006.

6. Ohsawa, Y.: "Discovering risky subduction zones of earthquake", Information Technology for Chance Discovery, 314-325, Tokyo-Denki University Press, 2005.

7. Ohsawa, Y.: "Visualizing relations between chances and surrounding events", Information Technology for Chance Discovery, 121-153, Tokyo-Denki University Press, 2005. 